\title{
Señderos
}

\section{Didáctica de la Educación Infantil. Reflexiones y Propuestas ${ }^{1}$}

\author{
Teaching children's education. Reflections and Proposals
}

\section{Autora: \\ Rosa Violante ${ }^{2}$}

\footnotetext{
1. Conferencia dictada en el II Congreso de Educación Infantil. Tecnológico de Antioquia Institución Universitaria, Facultad de Educación y Ciencias Sociales. Medellín, 16 y 17 de mayo de 2018.

2. Magíster en Didáctica con especialización en Didáctica General. Docente en la Universidad Nacional de San Martín - Argentina.

Correo electrónico: rosaviolante@gmail.com
}

En esta conferencia propongo presentar cinco reflexiones alrededor de la Didáctica de la Educación Infantil vinculadas con su estatus en tanto disciplina, con la caracterización de su objeto de estudio, con la tarea del docente, con las instituciones en las que se enseña y por último con el lugar que ha de ocupar en la formación docente. A partir de cada una de estas reflexiones planteo algunas propuestas a modo de líneas, para continuar pensando juntos la transformación de las prácticas docentes puestas en acción en el día a día del trabajo desarrollado con los niños y niñas menores de 6 años. Me siento parte de esta comunidad de aprendizaje y de una experiencia de construcción de conocimiento compartido, de la que hacemos parte todos los interesados en la educación inicial/infantil. 
Las cinco reflexiones que propongo son:

- Pensar la didáctica de la educación infantil como una didáctica específica y necesaria.

- Sostener una definición particular y propia de enseñanza cuando pensamos en enseñar a niños pequeños.

- Reconocer que el docente/educador como enseñante despliega diferentes acciones para asumir el compromiso político pedagógico de enseñar a los niños pequeños.

- Incluir los diversos formatos institucionales y modalidades alternativas a las que concurren niños y niñas pequeños bajo el concepto de "Escuelas Infantiles en sentido amplio" como los espacios en los que puede concretarse la educación infantil.

- Revisar el lugar central que tiene la Didáctica de la Educación Inicial en las propuestas de formación docente.

A modo de cierre y apertura, considero que estas reflexiones y propuestas forman parte de una línea de trabajo enfocada "Hacia la construcción del campo de la Pedagogía Infantil”, campo del que forma parte la Didáctica de la Educación Inicial, desde esta perspectiva.

\section{Primera reflexión: Pensar la Didáctica de la Educación Infantil como una didáctica específica y necesaria}

La Didáctica de la Educación Infantil es una didáctica específica en tanto reúne teorizaciones acerca de qué y cómo enseñar a los sujetos niños y niñas menores de 6 años. Las formas de enseñar han de responder a las posibilidades de aprendizaje que tienen los niños pequeños. En la educación infantil, las didácticas específicas por disciplinas o diferentes campos del conocimiento (didáctica de la Matemática, de Ciencias Sociales, etc.) se han de constituir solamente en aportes para enriquecer la Didáctica de la Educación Infantil. Desde esta perspectiva, el campo teóricopráctico de la Didáctica de la Educación Infantil constituye el referente central para pensar y organizar la enseñanza promoviendo una educación integral. Se reconocen como constitutivas del campo de la didáctica perspectivas con diferentes niveles de teorización (Ferry, 1991). Se incluyen:

- Teorías que no se proponen orientar el accionar, pero sí ofrecer argumentación para la toma de decisiones. Estas son los núcleos conceptuales de la didáctica de la educación infantil, por ejemplo: caracterización de las relaciones entre familias e instituciones, definición y caracterización del juego como actividad principal de los niños, entre otras (nivel de teorización 4) (Ferry, 1991).

- Repertorios de formas de enseñar que podrían reconocerse en los "Principios pedagógicos" o "Pilares" de la Didáctica de la Educación Inicial. Estas son orientaciones para la acción, suponen pautas, criterios argumentados para definir la acción del docente en relación con las diferentes variables de la situación de enseñanza. En el nivel praxiológico encontramos respuestas a: ¿Por qué? y ¿̇para qué? del ¿qué enseñar? y ¿cómo enseñar? Las respuestas a estas dos últimas preguntas constituyen el nivel técnico de teorización. El nivel de teorización praxiológico, que 
incluye el técnico, conforma los repertorios de formas de enseñar consideradas ética, política y pedagógicamente pertinentes para abordar el desafío de educar a los niños pequeños (niveles de teorización 2 y 3) (Ferry, 1991).

- Las prácticas de la enseñanza en acción. El diseño y la puesta en marcha de la enseñanza apelan al nivel de la acción, el cual supone también una reflexión en el curso de la misma acción. Se refiere al "saber hacer", a la reflexión "en” y "sobre” la acción (Schön, 1986). Además se nutre de las teorías y las interpela construyendo nuevas teorizaciones en función de las necesidades que surgen de las prácticas (nivel de teorización 1) (Ferry, 1991)33

3. Para ampliar la caracterización de los diferentes niveles de teorización de Ferry se transcribe a continuación un fragmento de la tesis de maestría de Rosa Violante: “[...] Ferry (1997) muestra en la escala de teorización propuesta qué lugar ocupa la teoría en la formación docente desde el nivel de las acciones hasta el nivel del conocimiento científico. En este sentido, plantea que puede teorizarse sobre las prácticas o bien sobre aspectos de la realidad que inciden en las prácticas. En su escala, Ferry reconoce cuatro niveles:

1. Nivel de la práctica o nivel del hacer

2. Nivel del conocimiento técnico

3. Nivel del conocimiento praxiológico

4. Nivel del conocimiento científico

Resulta importante hacer dos precisiones en relación con estos cuatro niveles. En primer lugar, desde el nivel 2 al nivel 4 aparece la expresión "conocimiento de”, es decir, explicitación de razones, referencia a algún tipo de construcción teórica. El nivel 1 se refiere al nivel de la acción. En segundo lugar, Ferry plantea que los tres primeros niveles representan diferentes grados de "teorización" con relación a las acciones prácticas. El cuarto nivel se diferencia de los otros tres porque se "despega" de las acciones, busca explicar condiciones, situaciones que inciden en las acciones prácticas, produce saberes libres de la preocupación por las acciones. En este último nivel, la teoría no responde a interrogantes sobre las acciones.

El primer nivel de la práctica o nivel del hacer se refiere al hacer empírico. Se responde con acciones frente a la pregunta ¿qué hacer? Se hacen presentes al actuar el conocimiento tácito (Polanyi, 1970), el conocimiento en la acción (Schön, 1986). No hay conciencia de tal conocimiento.

En el segundo nivel (técnico) se da un primer distanciamiento respecto de la acción, se produce un discurso sobre el hacer, un discurso empírico, dado que consiste en explicar pasos, proponer recetas para conseguir determinados resultados. Correspondería al primer nivel propuesto por Van Manen, el nivel técnico. Es un discurso técnico. Se construye con la respuesta a la pregunta ¿̇cómo hacer? Estos saberes son los saberes característicos que se necesitan para desarrollar un oficio. Los sujetos logran apropiarse de un "saber hacer" pero sin conciencia de las razones que justifican esas acciones. Este nivel, junto con el siguiente, correspondería a lo que Schön considera la "reflexión sobre la acción".

La diferencia entre el segundo y el tercer nivel, que a continuación desarrollaremos, consiste en que mientras en el segundo nivel se reflexiona sobre el qué hacer, y que supone dar cuenta de una racionalidad técnica (Habermas, 1971), en el tercer nivel se reflexiona sobre las razones, las finalidades y los fundamentos. Es una reflexión que puede dar cuenta de una racionalidad práctica, que en términos de Habermas supone un compromiso ético y social además de la consideración de las técnicas. En el tercer nivel (praxiológico) se construyen las respuestas a las preguntas ¿por qué hacer...? y ippara qué hacer? Los modelos del "saber hacer" se ponen en cuestión; se reflexionan y analizan cuáles son las implicancias de las acciones.

A nuestro entender, este tercer nivel correspondería al $2^{\circ}$ y $3^{\circ}$ niveles propuestos por Van Manen, es decir, se reflexiona tanto sobre los objetivos y principios de la práctica educativa como sobre los matices morales y políticos enmarcados en una perspectiva más amplia. Ferry explica que en este nivel "podemos empezar a hablar realmente de teoría porque no se trata de reproducir de manera idéntica recorridos o desarrollos que se pudieron haber practicado anteriormente sino que se va a pensar, a reflexionar sobre el sentido de estos trayectos" (Ferry, 1997, 78). Este nivel refiere al concepto de praxis, que no implica sólo el hacer técnico; la praxis demanda del docente "sabiduría práctica", capacidad para tomar decisiones en orden a los aspectos no sólo técnicos, sino éticos y sociales. Para Ferry, "la praxis no es sólo la práctica, la praxis es la puesta en obra de diferentes operaciones en un contexto dado que es necesario analizar y en el que habrá que tomar decisiones referentes al plan de ejecución de lo que se hace”. Este concepto de praxis explica el carácter ético, moral y político de la actividad docente mostrando cómo va más allá de un conjunto de pasos técnicos a seguir.

"En la praxis, el pensamiento y la acción (o la teoría y la práctica) guardan entre sí una relación dialéctica; deben entenderse como mutuamente constitutivas, en un proceso de interacción por medio del cual el pensamiento y la acción se reconstruyen permanentemente, en el seno del proceso histórico vivo que se manifiesta en toda situación social real. Ni el pensamiento es preeminente, ni lo es la acción" (Carr y Kemmis, 1986).

Finalmente, y en relación con el cuarto nivel, Ferry caracteriza al conocimiento científico como aquel que está conformado por las teorizaciones referidas a aspectos educativos, pero no directamente vinculados a la toma de decisiones para la acción. Son conocimientos que responden a preguntas tales como: ¿Cómo podríamos caracterizar la institución escuela? Perrenoud (1994) los llama saberes de referencia.

La reflexión y la teorización se realizan sobre las prácticas docentes, que son nuestro objeto de estudio y en cada uno de los niveles propuestos se abordan, a nuestro entender, diferentes dimensiones de dichas prácticas que no resultan excluyentes entre sí; por el contrario, toda práctica docente conlleva una dimensión técnica y práctica. 
La didáctica constituye el saber sobre el "saber hacer" relatado, escrito, tematizado, reflexionado. Por lo tanto, las perspectivas teóricas propias de la didáctica de la educación infantil recuperan teorías y prácticas muy valoradas por su carácter específico, construidas en el devenir cotidiano de la experiencia educativa desarrollada en las instituciones con niños menores de 6 años. Recuperan también parte de la historia pedagógica-didáctica de la educación inicial.

La Didáctica de la Educación Infantil es necesaria ${ }^{4}$ porque ha de explicitar y argumentar a favor de sostener formas de enseñar respetuosas de los niños. No todas las formas son legítimas. Es tarea de la didáctica pronunciarse en este sentido y explicitar las razones para que sean posibles los derechos de los niños a recibir educación, enseñanza que logre calidad e inclusión educativa. En segundo lugar, es necesaria en tanto ha de continuar con la tarea de pensar y analizar cuestionamientos en el proceso, siempre inacabado, de construir las formas de enseñar específicas.

Su tarea incluye proponer una organización de contenidos que atienda los modos más apropiados para ofrecerlos a los niños pequeños. De este modo se permite la democratización del conocimiento ofreciendo la posibilidad de que todos y todas puedan acceder a él. Permite llegar a todos y todas, los niños y las niñas, porque ayuda a encontrar las múltiples formas de lograrlo. Al mismo tiempo se ocupa de la reflexión sobre la función de la evaluación de los aprendizajes, y la incluye, para la re-visión de las propuestas de enseñanza en función de promover una educación integral.

Enseñar no es fácil, y enseñar a los niños pequeños es un desafío mayor. La didáctica de la educación infantil es necesaria en tanto ofrece múltiples saberes y conocimientos, expresados en los diferentes niveles de teorización que contribuyen a abordar la difícil tarea de enseñar.

\section{Primera propuesta en respuesta a la primera reflexión}

Una propuesta que expresa la especificidad y responde a la necesidad de contar con la Didáctica de la Educación Infantil la constituyen los "Pilares de la Didáctica de la Educación Inicial” (Violante y Soto, 2011). Los Pilares para organizar la enseñanza son principios que han de orientar las acciones docentes en el diseño, la puesta en marcha y la evaluación de la enseñanza y de los aprendizajes. En este sentido constituyen teorizaciones del nivel praxiológico y técnico. Responden a qué, cómo y por qué enseñar... Se presentan a modo de recomendaciones, orientaciones "irrenunciables", dado que constituyen las razones sobre las que descansan las formas de organizar la enseñanza respetuosa de las características particulares de los niños y niñas menores de 6 años. Los Pilares son:

4. Para ampliar estos planteamientos, véase Soto y Violante (2009). 


\section{Educación integral}

Los propósitos generales de la educación inicial se orientan a considerar que la formación personal y social y la alfabetización cultural, constituyen las dimensiones de una educación integral. Este enfoque integral se propone satisfacer las necesidades e intereses de los niños y niñas en forma armónica y equilibrada. Implica responder a las necesidades de afecto, cuidado, sostén, confianza y al interés por jugar, explorar, conocer el mundo y sus múltiples manifestaciones culturales. Proponer una educación integral es pensar en el derecho incuestionable a la educación, a acceder al conocimiento, a los saberes, las actitudes, los modos de actuar y pensar y las habilidades —expresados en los Núcleos de Aprendizaje/ en los contenidos, para todos los niños y las niñas. Este pilar ofrece orientaciones a la hora de seleccionar que enseñar.

\section{Globalización}

Las propuestas de enseñanza que se ofrecen a los niños y niñas pequeños han de responder al principio de globalización. Esto significa presentar situaciones totalizadoras y evitar la fragmentación a la que se tiende cuando se seleccionan contenidos para enseñar desgajados de las propuestas o de los contextos de los que forman parte. Los organizadores de la enseñanza propios de la educación inicial (proyectos de juego, proyectos para conocer el ambiente, la vida cotidiana, las experiencias estéticas y la multitarea) (Violante y Rodríguez, 2017) se caracterizan por constituirse en propuestas - recortes "globales" o con una estructura, o propuestas totalizadoras que resultan significativas para los niños y las niñas. Desde las propuestas se apela a la inclusión de los diferentes contenidos de los campos de experiencias que sean pertinentes de abordar para cada caso. Se nuclea o aglutina aquello que guarda sentido con determinado proyecto de juego, o con la oferta de multitarea, o con el organizador de la enseñanza que lleve adelante el docente.

De este modo se posibilita que todos los niños y las niñas accedan a la construcción de conocimientos a partir de una intencionalidad docente, que enfatiza el derecho a la democratización de los saberes como patrimonio de todas y todos. Este pilar constituye una de las argumentaciones a partir de la cual se proponen los organizadores de la enseñanza ya mencionados como “configuraciones didácticas" propias de la educación inicial.

Centralidad del juego (Violante, 2011)

El juego resulta un modo de expresión singular, un derecho inherente a la infancia (ver Violante, 2011). Expresa una forma peculiar y gozosa de manifestarse, de conocerse a sí mismos, conocer a otros y otras, a su comunidad y al mundo. Considerar la centralidad del juego implica otorgarle un lugar central en el ámbito escolar, dado que para los niños y niñas resulta un modo sustantivo a través del cual aprenden en forma gratificante, intrínsecamente interesante y expandiendo las fronteras de sus repertorios de vida. Jugar en el contexto escolar requiere que los docentes elaboren propuestas lúdicas, que comprometan la distribución de los tiempos, los espacios, materiales, los grupos. De la misma manera, se ha de poner a disposición la posibilidad 
de jugar diferentes juegos que han de estar presentes en el devenir cotidiano de todas las salas de la escuela infantil.

La centralidad del juego involucra pensar las propuestas en clave de juego a sabiendas de que existen otras actividades también legítimas, que no son juegos, como las experiencias estéticas, u otras propuestas pertenecientes a distintos campos de experiencias que promueven también aprendizajes significativos. El juego constituye un contenido de alto valor cultural, y al mismo tiempo un medio para enseñar otros contenidos-núcleos de aprendizaje de otros campos de experiencias comprometidos en los verdaderos juegos. Estos tres primeros pilares (educación integral, globalización y centralidad del juego) responden a las preguntas de para qué y qué enseñar. Se propone ofrecer los saberes y contenidos culturales con el propósito de promover la educación integral. Los pilares que siguen se pronuncian sobre cómo enseñar.

\section{Multitarea}

La multitarea (Violante, 2013a) ha de constituirse en una de las modalidades privilegiadas para organizar la enseñanza. Propone ofertar en simultáneo varias alternativas de actividades y/o juegos, entre los que espontáneamente los niños y las niñas pueden optar. Supone una organización del espacio particular, zonificado; conlleva la conformación de pequeños grupos de niños y niñas, hecho que aumenta y enriquece la interacción entre ellos. Permite el respeto por los tiempos individuales de aprendizaje y una intervención docente más particularizada, sin perder de vista el desenvolvimiento de la actividad en el grupo total. Al proponer diferentes actividades, se promueve la educación integral.

\section{Ambiente alfabetizador}

Se caracteriza un ambiente alfabetizador cuando implica considerar que el espacio ha de ser mediado o intervenido pedagógicamente por el docente que enseña. La construcción de escenarios es condición básica para poder llevar una enseñanza que ofrezca contextos de vida enriquecedores para los niños y niñas. Las formas convencionales que suponen un espacio con mesas y sillas exclusivamente y actividades dirigidas al grupo total, para que al mismo tiempo todos realicen las mismas acciones y del mismo modo, resultan escenarios empobrecidos, en los que se dificulta armar dinámicas de trabajo en las que se promueva la autonomía y la atención individual de cada niño y cada niña. La oferta de materiales diversificados y polivalentes conforma una parte constitutiva de la construcción de un ambiente alfabetizador. Un ambiente alfabetizador supone considerar que el espacio enseña, si es diseñado intencionalmente por el docente.

\section{Tiempos flexibles y diversos}

La organización flexible de los tiempos se propone como respuesta a la necesidad de un diálogo permanente entre los tiempos personales, grupales e institucionales. Compromete tiempos de actividades diversas: cotidianas, de tiempos intermedios (esperas, por ejemplo), grupales, individuales, electivas, entre otras. En el tiempo 
total de la jornada escolar, para las niñas y niños más grandes han de prevalecer situaciones de enseñanza en clave de juego y multitarea.

Los tiempos propuestos para las diferentes actividades también han de considerar las edades y posibilidades de los niños y las niñas, sus umbrales de tolerancia atencionales y el tipo de juegos y tareas que se les han de ofrecer. Las "rutinas"s estables (en tanto actividades cotidianas no automatizadas) resultan organizadores estructurantes de las experiencias cotidianas, las hacen previsibles, contribuyen a promover la autonomía, la seguridad y la confianza en los niños y niñas.

Formas de agrupamientos diversos y alternados

La conformación de agrupamientos de los niños dependerá de la variedad de tareas. Resulta rico y dinamizador pensar en la diversidad y alternancia proponiendo las actividades para desarrollar en pequeños grupos, homogéneos y heterogéneos (según los propósitos docentes), grupos de edades integradas y salas multiedad, como alternativas positivas y favorables que enriquecen y amplían el intercambio, la participación de los niños y niñas en contextos de vida que resultan beneficiosos. Estas alternancias enriquecen el intercambio, la autorregulación de las interacciones entre niños y niñas con diferentes posibilidades e intereses, y promueven el pensar juntos, la conquista de valores como la solidaridad, el trabajo con otros, el respeto por los demás y por las propias necesidades en el desarrollo de las diferentes actividades

La experiencia directa y el planteo de situaciones problemáticas, actividades que consideran a los niños y las niñas activos protagonistas en la construcción de sus aprendizajes

La experiencia directa y el planteo de situaciones problemáticas en las que participan las niñas y los niños constituyen una de las formas de promover la construcción de conocimientos relacionados con el ambiente, con proyectos de juego y con determinados escenarios. Estas propuestas promueven procesos de aprendizaje significativos. El niño y la niña actúan directamente sobre los objetos, observan cómo reaccionan y construyen así el conocimiento acerca de sus propiedades; visitan los espacios sociales y participan en su devenir cotidiano de tal modo que han de iniciarse en la indagación del ambiente social; buscan alternativas posibles frente al planteo de situaciones problemáticas. Todas estas formas de presentar las actividades promueven la participación activa y el protagonismo de los niños y las niñas.

\section{Familias, comunidad e instituciones comparten la enseñanza}

El trabajo docente ha de incluir el diseño de acciones sistemáticas de trabajo conjunto con las familias. Resulta beneficioso que la institución y los docentes logren generar espacios de participación y diálogo para acompañar el crecimiento de los niños y las niñas en forma complementaria y enriquecida. El hecho de sostener lazos de confianza establecidos con las familias y la interacción constructiva con la comunidad comprometen una escuela infantil, abierta, plural e integral.

5. Rutinas (se toma de los aportes de Zabalza [1996]) pero no comprendidas como rutinización en estructuras rígidas de horarios y de distribución espacial, sino como rutinas constructivas, entendidas como ciertos rituales, secuencias de acciones que se reiteran y se pueden anticipar para generar un sentimiento de seguridad por el porvenir cotidiano. 
El docente, artesano de la enseñanza, diseña y anticipa sus actuaciones como "acompañante afectivo, figura de sostén, otro significativo" y como "mediador cultural"

El docente enseña compartiendo expresiones mutuas de afecto, ofreciendo disponibilidad corporal, realizando acciones de manera conjunta, acompañando con la palabra el juego, reiterando en forma recursiva las propuestas, entre otras formas de enseñar específicas y particulares para los niños y niñas menores de 6 años.

\section{El docente evalúa la enseñanza y los aprendizajes para comprender y actuar}

La evaluación, entendida como el proceso de comprensión y análisis por parte de los actores involucrados en la experiencia educativa compartida, permite revisar, ajustar y realizar un seguimiento de la enseñanza y de los aprendizajes grupales e individuales de los niños y niñas, para orientar la toma de decisiones que contribuyan al logro de los propósitos esperados.

\section{Segunda reflexión. Sostener una definición específica y propia de enseñanza cuando pensamos en enseñar a niños pequeños}

Una definición de enseñanza dirigida a niños y niñas desde el nacimiento hasta los 6 años tiene que responder a las particulares formas de aprender de los niños pequeños, por lo tanto, atender a formas de enseñar específicas de la educación inicial. La enseñanza en el nivel inicial exige una definición propia ${ }^{6}$, se refiere a todo el conjunto de acciones ${ }^{7}$ que despliega el adulto para que los bebés y los niños crezcan en un ambiente seguro y enriquecedor, que potencie el desarrollo en sus diferentes dimensiones (física, afectivo-social, cognitiva, lingüística, artística, etc.), al mismo tiempo que se les ofrezca como un "universo a descifrar, construir, transformar". Enseñar en el nivel inicial supone acompañar al bebé y al niño en la construcción de los significados culturales que portan los objetos, las acciones, los gestos, los modos de actuar, los festejos, las costumbres. Supone también ofrecer experiencias de observación, exploración y experimentación para ampliar y enriquecer el conocimiento de las características del mundo físico y natural.

Enseñar en el nivel inicial es dar: conocimiento y afecto, confianza, calidez, ternura, cuidado; es acunar desde los primeros años con "brazos firmes pero abiertos" que ofrezcan seguridad y posibilidad de autonomía; es alertar sobre los peligros, es mostrar el mundo y cómo andar en él, es saber retirarse cuando el bebé y el niño manifiestan que pueden resolver por sí solos. Es necesario mencionar que en el caso del trabajo en las primeras sesiones, la enseñanza ha de ser un modo de asumir la crianza en el sentido de ofrecer alimento y cuidado, nutrir para permitir crecer ${ }^{8}$. Nutrir implica no

\footnotetext{
6. Se transcriben fragmentos del documento: Violante, R. (2001). Enseñanza en el Nivel Inicial I y II. Disponible en http:// studylib.es/doc/6335702/ense\%C3\%B1anza-en-el-nivel-inicial-1-y-2

7. Al hablar de "las acciones docentes" incluimos las realizadas antes, durante y después del desarrollo de la actividad propiamente dicha llevada a cabo junto con los niños. Es decir, planificar es una de las acciones que despliega el docente para lograr que los niños aprendan, de igual modo que las reflexiones posteriores al desarrollo de la actividad.

8. En las primeras secciones tiene una importancia fundamental y ocupa gran parte del tiempo de la jornada, lo que no significa que no se continúe con la crianza en las edades más avanzadas.
} 
sólo ofrecer la comida, el biberón, etc., nutrir se refiere a ofrecer conjuntamente con el biberón la contención afectiva, el sostén, los brazos que ofrecen la calidez humana que hacen del momento de la alimentación una experiencia plena que se desea repetir porque produce bienestar físico y emocional. Tomando una definición genérica de enseñanza (Fenstermacher, 1988) como una actividad intencional que implica una relación particular entre, por lo menos, dos personas, en la que una de ellas posee un saber, experiencia y conocimiento que quiere transmitir a otro sujeto, vemos que en el nivel inicial se enseña, pero la modalidad en que se transmite a los bebés y niños la cultura, las habilidades, la necesidad de autonomía y la seguridad para confiar en los otros adquiere formas particulares.

\section{Segunda propuesta en respuesta a la segunda reflexión}

Considerando la necesidad de pensar en una definición particular cuando se trata de enseñar a niños pequeños, compartiré a continuación las categorías teóricas construidas en diversas experiencias que he transitado como profesora investigadora y formadora de docentes. Se trata de una caracterización de diversas formas de enseñar específicas propias de la educación inicial ${ }^{9}$. Se proponen ocho formas de enseñar:

Enseñar... Expresando y compartiendo los afectos.... Arrullos, abrazos, emociones, miedos, alegrías

Ser parte de expresiones mutuas de afecto son formas cotidianas de acompañar la construcción de vínculos de confianza y de afecto con los otros. Es así que se ayuda a sentir que el mundo es un lugar seguro, grato, que vale la pena explorar. También se les enseña a confiar en el mundo y en ellos mismos, expresándoles cariño y reconocimiento frente a sus logros, disfrutando el cantar juntos las nanas, o canciones que se les cantaban a los docentes cuando eran pequeños, y cantar o tararear canciones provenientes de las familias.

Se enseña, se transmite el legado cultural, los bienes simbólicos de la cultura, se preservan tradiciones de las comunidades, de los pueblos originarios, de otras comunidades provenientes de países limítrofes o de otras procedencias. Desde la escuela infantil y la educación integral, se materializan también los principios de inclusión y democratización, recuperando el acervo popular de los pueblos, rescatando retazos de la memoria colectiva, desempolvando el caudal cultural que atesora la diversidad de los ancestros que constituyen la nacionalidad.

Enseñar... Ofreciendo el sostén y la disponibilidad corporal

Con los más pequeños, el cuerpo puede abrazar, sostener, acunar; se convierte por momentos en un "caballito gris" en el que los chicos se "suben", se sientan sobre las piernas de los docentes para jugar y galopar. También el cuerpo puede convertirse en el mejor "almohadón” para dormirse confiado y sereno. Corresponde que el docente

9. La síntesis se ha elaborado a partir de dos escritos anteriores. Véanse: Violante (2006) y Soto y Violante (2005). 
se ofrezca con su cuerpo disponible, sentándose en el piso con ellos, o convirtiéndose en muchas ocasiones en soporte —o sostén - para que se animen a ponerse de pie, o en una suerte de "colchón humano" donde recostarse para descansar, alimentarse, sentirse protegido, querido. Con los niños más grandes se puede jugar a las palmaditas, entrechocando las manos con las de los niños y niñas, mientras se recitan rimas o se realizan juegos de fórmulas. De este modo, se va viendo que se ofrece disponibilidad corporal, de sensibilidad corporal y gestual; se enseña jugando, se enseña a confiar en las propias posibilidades de las niñas y los niños, se alienta la autoestima, se genera un clima de alegría compartida.

Enseñar... Mirando a las niñas y niños, con interés y atención. Observar

Se propone observar para dar seguridad, confianza y acompañar respetuosamente los procesos exploratorios y lúdicos. Cuando se los está mirando, si se lo hace con interés y atención, se le está enseñando que aquello que van haciendo es importante para los docentes. Esto favorece que ellos sientan confianza en sus posibilidades y se animen a explorar y descubrir el mundo.

Observar para reflexionar acerca de cómo se ha de actuar, para interpretar las necesidades, demandas, o "pedidos” explícitos o implícitos que expresan los bebés, así como las niñas y niños. Los docentes interpretan el llanto, los gestos, determinadas vocalizaciones y balbuceos, miradas, movimientos, las expresiones de molestia, las sonrisas, la alegría, y deciden en la acción cómo ofrecerle a cada una de estas manifestaciones un especial acompañamiento y atención. En el caso de niñas y niños mayores de dos años, la observación atenta del actuar en el desarrollo de las juegos y actividades permite encontrar las formas de intervenir para enriquecer éstos, ampliando las oportunidades de juego, aportando información para resolver situaciones, mediando ante conflictos, entre otras variadas formas de participación docente.

\section{Enseñar... Realizando las diversas acciones en forma conjunta}

Hay muchas situaciones que los bebés, niñas y niños no pueden realizar si no es en conjunto, con el apoyo sensible de los adultos, por eso los adultos enseñan realizando acciones junto con los niños o conjuntamente. Es decir, en un primer momento, "lo hacemos por ellos, junto a ellos". Por ejemplo: cantar una canción; reiterar un poema; realizar un juego con las manos; mirar el mundo "de parados", se les "presta" la postura erguida, en tanto posibilidad de estar "parados" con el adulto, caminar con ellos a "upa”. De esta manera, ven el mundo, “como si” caminaran... Se canta el juegocanción Qué linda manito, y poco a poco el niño va repitiendo, junto con el adulto, algunas partes. Por esto, a medida que el niño va imitando y realizando las acciones, se lo va dejando solo para que logre, por ejemplo, cantar en forma autónoma, o de manera independiente. También con los niños más grandes, por ejemplo, se enseña a jugar un juego con reglas convencionales, como es el caso del "Ta-te-tí", o "La casita robada” con cartas. Adultos-niñas-niños, lo juegan conjuntamente muchas veces; al decir de Patricia Sarlé, se les "presta conciencia” acerca de los secretos del juego, "los guiños y sus mañas". A medida que lo juegan, lo van aprendiendo, hasta que 
lo pueden hacer con otros chicos y chicas, sin necesidad de la presencia del adulto. Como se ha tratado, más lo juegan, más lo comprenden, mayor dominio y autonomía van adquiriendo. Cuanta mayor autonomía logran, mayor libertad adquieren. Para el logro del dominio y la autonomía hace falta un docente que haya enseñado, vaya "haciendo juntos", o bien, que enseñe a jugar, jugando (P. Sarlé). Tal como lo plantea Bárbara Rogoff, al desarrollar la conceptualización de "participación guiada" y "traspaso paulatino del control de la situación”, es evidente que el adulto atento, que sabe observar las posibilidades de acción de los bebés y niños, logra retirarse a tiempo y favorecer la asunción de la responsabilidad de la acción por parte del niño, en el momento en que cuenta con las condiciones para asumirlo.

Enseñar... Construyendo lugares, espacios para jugar, enseñar y aprender. Enseñar construyendo escenarios implica ofrecer un ambiente alfabetizador

Esta forma de enseñar se refiere a la consideración del espacio como dispositivo para organizar la enseñanza. Estos conceptos se han desarrollado en detalle en apartados anteriores.

Enseñar armando un repertorio, o itinerario en el que resulte posible la reiteración, la repetición Repetir es dar oportunidad de volver a hacer, de que el niño y la niña recuperen memoria acerca de lo construido, y logren progresar en su sistema de interpretación o en las habilidades que despliega, de manera tal que vaya adquiriendo un paulatino dominio y afianzamiento de los aprendizajes. Para los más pequeños, las formas de enseñar-jugar, de jugar-aprender, necesitan ser reiteradas, repetidas, para que el aprendizaje, que es un proceso individual, propio de cada uno, se vaya dando en los tiempos que cada niño necesita. La reiteración favorece que las niñas y niños vayan comprendiendo el formato de los juegos, por ejemplo, que se vayan apropiando de él. En la medida en que se les brindan muchas oportunidades de reiteración, se da la posibilidad de que el niño y la niña lo puedan ir haciendo solos, cada vez con la menor ayuda posible. Cuanto más domina, más libre y creativo puede llegar a ser. Repitiendo exploraciones, distintos formatos de juegos, canciones, abrazos, rituales, el niño disfruta y se manifiesta cada vez más autónomo en sus juegos y acciones cotidianas. En el caso de los niños más grandes, la reiteración sigue resultando necesaria. Se reitera, de la misma manera hasta que la propuesta o situación planteada se va ampliando, al irse agregando otros modos de enriquecer la situación original. A medida que los niños lo repiten, se van familiarizando con aquello que se les propone, van comprendiendo cada vez mejor y haciendo o participando de manera más dinámica, más plena.

Enseñar... Proponiendo experiencias directas y planteando situaciones, preguntas, problemas Esta forma supone enseñar planteando problemas y/u ofreciendo situaciones, experiencias directas que impliquen un reto, o desafíen a los niños y niñas a pensar, comparar, expresarse, relacionar, sacar conclusiones. Muchas veces haciendo algunas preguntas que guarden sentido con la tarea o juego que llevan a cabo, se puede ayudar a que los niños más grandes se sientan invitados a analizar, conocer o comprender el mundo que los circunda. En las búsquedas de las respuestas a estas preguntas van aprendiendo diferentes saberes, van construyendo nuevos conocimientos, se van 
apropiando de múltiples aprendizajes en los que participan diferentes campos de experiencias y contenidos.

\section{Enseñar... Nombrando lo que hacemos... hablando}

La palabra se hace presente para expresar afecto, interés por lo que hace el niño, reconocimiento por sus logros, atención y preocupación, sirve para preguntar, indagar, valorar... Es importante hablarles a los niños pequeños de modo completo, aun cuando aparentemente no comprenden en detalle cada palabra, porque siempre registran el sentido y significado total del mensaje. En el devenir cotidiano en grupos de niños y niñas más grandes, dar espacio a la palabra contribuye a construir sentidos y significados, resulta una actitud central. Hablar, escuchar, intercambiar, compartir ideas, en pequeños grupos, entre niños y niñas, entre niños-niñas y adultos. A través de la palabra se transmite el sentido social y cultural que tienen ciertos modos de actuar, como así también el uso social de los objetos. La palabra encierra y transmite los sentidos sociales de las acciones y del uso de los objetos, la palabra explica, indica, "abraza”, sanciona, ampara, valoriza, reprueba, informa, alienta, alerta, expresa, brinda alegría y confianza. Parafraseando a Gianni Rodari: El uso total de la palabra para todos y todas, no para que todos sean artistas (literatos), sino para que ninguno sea esclavo. Los arrullos, las palabras que buscan inducir al sueño, transmiten tibieza, ternura, musicalidad, placer por escuchar. Las palabras, al igual que los brazos, acunan.

El lenguaje se enseña y enseña otros aspectos hablando junto con los niños-niñas y para los niños-niñas. A partir de estas ocho formas de enseñar es interesante reflexionar acerca de cuáles resultan más apropiadas según el contenido o núcleo de aprendizaje que se quiera enseñar. A modo de ejemplo, para enseñar a jugar juegos de exploración de propiedades de los objetos / juegos de construcción, principalmente se proponen escenarios con diversos materiales para favorecer la actividad exploratoria autónoma de los niños. En cambio, para enseñar un juego tradicional como el "veoveo", es la realización conjunta y la repetición del formato lo que permitirá que el niño se apropie de este juego y luego pueda jugarlo en forma autónoma.

\section{Tercera reflexión. Reconocer que el docente/educador despliega diferentes acciones como enseñante para asumir el compromiso político-pedagógico de enseñar a los niños pequeños}

El docente diseña, planifica, piensa y propone "configuraciones didácticas" en tanto formas particulares de articular el qué y el cómo enseñar sostenidas en las razones de enseñar (el para qué y el porqué). Cuando se piensa en el qué enseñar en las instituciones educativas dirigidas a los niños pequeños, se debe proponer ofrecer una educación integral. La misma supone favorecer y enriquecer el desarrollo personal y social (enseñando autonomía, trabajo con otros, constitución de la identidad, etc.) y promover procesos de alfabetización cultural. Esto último implica acceder al mundo simbólico del juego, los lenguajes artístico-expresivos, las producciones culturales de las comunidades, costumbres, formas de danzas, historias, conocimiento del ambiente social y natural, etcétera. 
El cómo enseñar nos remite a las formas de enseñar propias de la educación inicial ya expuestas en el apartado anterior. Se entiende que estas configuraciones didácticas se constituyen en una forma de concretar el derecho a acceder a una educación de calidad, y por lo tanto en oportunidad de verdadera inclusión. No es suficiente con que los niños asistan a las instituciones, en ellas además han de ofrecerse oportunidades para aprender los contenidos correspondientes a cada eje de experiencias, área curricular, dimensiones, etc., para recibir educación integral. Este planteo de cómo lograr calidad e inclusión lo analizaremos en la cuarta reflexión.

En relación con los modos de actuación del docente, las diferentes formas de enseñar resultan unas más adecuadas que otras según el contenido que se vaya a enseñar. Por otra parte, consideramos de interés reflexionar acerca de no polarizar en falsas antinomias el enseñar por medio de escenarios, disponiendo materiales como una forma hoy reconocida y oponerlos a la presentación de consignas más precisas que indican modos de resolución de determinadas situaciones. Pensamos que para el caso de los juegos de construcción puede resultar tan valioso proponer materiales y dejar que autónomamente el niño explore, pruebe y construya, como también ofrecer 'modelos' para construir siguiendo algunos pasos, no como la única forma de proponer los juegos de construcción, sino como una entre tantas propuestas con diferentes grados de direccionalidad externa ejercida por parte del docente.

A modo de ejemplo, en el caso del juego dramático, es tan válido proponer armar un escenario que represente el almacén y proponer jugar a comprar y vender, como también dejar dispuestos elementos no estructurados como cajas, telas, tubos, y otros objetos para que surja el tema de la dramatización de parte de los niños. Ambas propuestas contribuyen y enriquecen el desarrollo personal y social y la alfabetización cultural, porque ayudan a que los niños aprendan los modos sociales de ser y estar en diversos contextos, en la calle, el almacén, el colectivo, el teatro, la casa, el museo, etcétera. Se propone incluir situaciones de juego dramático con diferentes grados de direccionalidad externa. En general, en el juego en el patio o en el rincón de dramatizaciones suceden los juegos dramáticos cuyas temáticas son propuestas por los niños, y en otras situaciones se suman las temáticas y los escenarios dispuestos y propuestos por los docentes.

\section{Tercera propuesta en respuesta a la tercera reflexión}

Proponemos diseñar la enseñanza en términos de configuraciones didácticas propias de la educación inicial. Estas son formas de planificar y diseñar la enseñanza que atienden a los principios enunciados en los Pilares. ¿Qué se propone diseñarplanificar periódicamente?: Propuestas de juego, propuestas para conocer el ambiente y la vida cotidiana, experiencias estéticas y multitarea. Estos organizadores suponen considerar como dispositivos ‘claves' el tiempo, el espacio y los grupos.

Consideramos que los modos de diseñar la enseñanza se deben plantear desde la perspectiva de una "nueva agenda" constituida por estas categorías didácticas. Más allá de los planteos clásicos de objetivos, contenidos, actividades y materiales 
nos interesa especialmente que el docente asuma el desafío de enseñar armando escenarios, esto supone, como ya se ha explicitado, tomar el dispositivo espacio y confiar en que, a partir de un buen diseño de éste con oferta de diversos materiales, propuestas y/o problemas que estarán al alcance y con posibilidad de ser elegidos por los niños y niñas en forma autónoma.

En cada espacio o zona pensados se disponen materiales con cuya interacción los niños podrán aprender diversos contenidos específicos que contribuyen a lograr una educación integral. La idea es principalmente ofrecer espacios simultáneos todos desafiantes, interesantes, con ofertas que inciten al aprendizaje de contenidos de los diversos ejes de experiencias y dimensiones del aprendizaje. La multitarea, principio pedagógico, pilar y a la vez organizador privilegiado de la enseñanza para los niños pequeños, ofrece posibilidades de elección, trabajo en pequeño grupo, lo que implica mayor interacción y respeto por los tiempos individuales y grupales en forma simultánea, logrando un clima cotidiano de interés, trabajo, participación y aprendizaje significativo en el cual el docente puede intervenir en los subgrupos de manera más tranquila, pudiendo escuchar u observar a cada uno en su hacer y al grupo en el devenir cotidiano.

Cuarta reflexión. Incluir a todos los diversos formatos institucionales y modalidades organizativas alternativas bajo el concepto de "Escuelas infantiles en sentido amplio"

Todas las instituciones, todas las modalidades alternativas y formatos organizacionales que atienden y educan a niños pequeños han de cumplir finalidades educativas no solo de cuidado, por lo tanto, entendemos que constituyen diversas formas de "hacer" y "ser" escuelas infantiles en sentido amplio. Escuela porque se enseña, infantil porque asisten niños menores de 6 años. Es una forma de lograr verdadera inclusión y justicia social. Un lugar abierto a todos y todas las niñas y niños.

\section{Cuarta propuesta en respuesta a la cuarta reflexión}

Partimos de afirmar que, desde nuestro punto de vista, las escuelas infantiles han de ser las instituciones que se ocupen de educar a los niños de 45 días a 5 años inclusive, es decir, las instituciones para concretar la educación inicial hoy. "El nombre Escuelas Infantiles surge de esta nueva voluntad para adecuar las organizaciones de educación inicial a las necesidades actuales y a la evolución alcanzada por el conocimiento.

\footnotetext{
"Escuelas" es por la intencionalidad de recuperar el papel de la escuela y valorarlo, al mismo tiempo que definir explícitamente el carácter educativo de la institución; "infantil” porque sin duda tiene características muy específicas debido a la edad de los niños que constituyen su población.

(Penchansky y San Martín de Duprat, 1995, p. 38)
}

Pensar en las Escuelas_Infantiles: “dos puntos”, significa pensarla como institución en sentido amplio, es decir, explicitando e incluyendo todas las formas y modalidades 
organizativas. Así podemos pensar como Escuelas Infantiles (“dos puntos”), jardines de infantes, jardines integrales, jardines maternales, centros de desarrollo infantil, salas cuna, salas de juego, centros de crianza, centros de primera infancia, comedores escolares con extensión horaria, ludotecas, entre otras modalidades diversas. Se propone también la idea de pensar en Escuelas Infantiles "por crear". Cuando planteamos "por crear", quiere decir en respuesta a demandas y/o necesidades particulares, pero siempre interpretadas desde el marco de las normativas y regulaciones nacionales, provinciales, jurisdiccionales y/o las construidas a partir de aportes de especialistas en educación inicial. Entonces pensamos en instituciones que respondan a necesidades, demandas, y posibilidades regionales, familiares y comunitarias, es decir, en formatos aún "por crear". Se incluyen en esta categoría las instituciones de gestión estatal y privada pertenecientes a organizaciones diversas, gremios, sindicatos, etcétera.

En todos los documentos y escritos actuales, con la nominación escuela infantil se hace referencia sólo a las instituciones que tienen a su cargo a niños de 45 días a 5 años y en las cuales se reconocen seis secciones (sala de bebés, deambuladores, salas de 2, 3, 4 y 5 años), dentro de la educación inicial como primer nivel del sistema educativo ${ }^{10}$. Pensar las escuelas infantiles en sentido amplio, refiere a una acepción y nominación presentadas aquí que se proponen para incluir bajo la misma nominación todas las diferentes modalidades y formatos organizativos diversos que hoy existen y a los que asisten los niños menores de 6 años, apelando a que todas tengan una dependencia, supervisión o trabajo compartido con el área de educación.

En cambio, pensar en "punto y aparte" separa instituciones diferentes para circuitos y contextos distintos. Por un lado, las escuelas infantiles, jardines de infantes y jardines maternales pertenecientes al ámbito de la educación formal, por lo tanto dependientes de los ministerios de educación y "punto y aparte": centros de desarrollo infantil, centros de primera infancia, instituciones que atienden a niños menores de 4 años y a veces también se incluyen las salas de 5 años que pertenecen a otros ámbitos, y dependen de los ministerios de desarrollo social, de sindicatos y otras organizaciones sociales.

¿Cuál es la condición que han de cumplir todas las instituciones para ser consideradas escuela infantil?

Desde nuestro punto de vista, la condición irrenunciable es el carácter educativo de sus acciones, como el modo de concretar el derecho que todos los bebés, niños y niñas pequeños tienen a recibir educación de calidad. Calidad e inclusión conforman las dos caras de la misma moneda y se hacen presentes en la concepción de escuelas infantiles en "sentido amplio" que se quiere defender en el presente escrito. Logramos inclusión real construyendo de manera "situada" todos los diversos formatos que cada región, comunidad o grupo necesiten para sus niños pequeños. Es decir, pensamos que

10. Para ampliar se puede consultar Bialostocki et al. (2016). En el ítem III del capítulo 2, "Un poco de historia acerca de las instituciones de Educación Inicial”, se desarrolla con más detalle cuándo surgen las escuelas infantiles en Argentina. 
entre educación y las diferentes organizaciones sociales es fundamental reconstruir diálogos, acuerdos y compromisos compartidos.

Reconociendo la potencialidad educativa en todas las diversas modalidades y formatos institucionales existentes hoy, se facilita la posibilidad de que sean muchos (con la intención y la posibilidad de que sean todos y todas) los/as que puedan acceder a la educación. Calidad hoy implica que en todas las instituciones se enseñen contenidos desde la singularidad que estos adquieren, cuando pensamos en niños pequeños. Nos referimos a los quehaceres de la vida cotidiana, el juego, las prácticas del lenguaje, las experiencias estéticas y todos aquellos referidos a la constitución de identidad y la vida con otros. Estos son por excelencia los contenidos a enseñar en los primeros años en las diversas escuelas infantiles "en sentido amplio", los cuales se hacen presentes en el devenir del tiempo cotidiano. De este modo se alienta a las familias y a los niños pequeños a participar de contextos de vida enriquecedores que amplíen sus universos culturales. Es necesario que se lleve adelante un proyecto educativo institucional a cargo de docentes y educadores especializados para hacerse cargo de uno de los desafíos más comprometidos de nuestro tiempo.

Entonces, al pensar en Escuelas Infantiles en "sentido amplio" en la Educación Inicial (E. I. en "sentido amplio" en la E. I.), estamos pensando en instituciones educativas diversas, con formatos organizativos y modalidades diferentes para atender a necesidades ${ }^{11}$ y características regionales, comunitarias y familiares particulares; todas las situaciones han de recibir una respuesta educativa, y el Estado ha de ser garante del derecho de todas y todos los niños y niñas desde 45 días a 5 años inclusive a recibir educación.

\section{Quinta reflexión. Reflexionar acerca del papel central que tiene la didáctica de la educación inicial en la propuesta de formación docente}

La didáctica de la educación inicial/infantil como parte del campo de la formación específica (en la carrera de formación docente), es eje articulador de lo trabajado en otras instancias curriculares. Toma los aportes también de algunas disciplinas del campo de la formación general para reformularlos en función de sus particulares problemas propios de la enseñanza y su organización en instituciones que atienden a niños menores de 6 años. También es fuente y referente para enriquecer e 'iluminar' las reflexiones sobre las propias prácticas docentes puestas en marcha en los talleres del campo de la formación práctica profesional.

En el desarrollo de la instancia curricular didáctica de la educación inicial se llevan a la práctica los principios pedagógicos, los pilares que orientan la organización de la enseñanza. En el desarrollo de los talleres de las prácticas se vuelven a abordar los mismos temas, pero se ordenan y desarrollan a partir de los problemas que se

11. Si bien las instituciones muchas veces nacen para atender necesidades, de las familias, madres que trabajan, etc., no debe dejar de considerarse que los niños, en tanto portadores del derecho a la educación desde el nacimiento, han de asistir a las instituciones que se constituyan en espacios de protección integral de derechos. 
presentan en las prácticas de enseñanza, de la necesidad de recurrir a ellos para hacerlas más comprensibles y poder tomar decisiones mejor informadas.

\section{Quinta propuesta en respuesta a la quinta reflexión}

A partir de estas reflexiones que señalan el lugar “clave” que ocupa la Didáctica de la Educación Infantil en el recorrido formativo de la carrera de formación de docentes, presentamos a continuación algunas líneas para poner en marcha lo señalado. Como hemos planteado es necesario "poner en diálogo" lo que se trabaja y/o aborda en los espacios curriculares y las prácticas que suceden en las instituciones infantiles. También "poner en diálogo" lo trabajado en los distintos espacios curriculares de la formación docente. Para concretar este “doble diálogo" proponemos, a modo de ejemplo:

El diálogo entre didáctica de la educación infantil y otros espacios de la formación docente puede iniciarse a partir de un análisis y una definición conjuntos de la bibliografía que cada profesor tome en el dictado de su curso. Así podemos tomar bibliografías de referencia diversas sin que se reiteren para ampliar el marco referencial. Por otra parte, también pensamos que puede ser enriquecedor tomar la misma bibliografía, pero efectuar sobre ella diversas miradas desde las perspectivas de cada materia, miradas concurrentes: ¿qué nos dicen los autores para analizar proyectos de ambiente, o propuestas de arte o actividades cotidianas?

En el campo de las prácticas, retomar y volver a consultar, apelar a marcos teóricos ya vistos en las diversas materias para hacer más inteligibles las prácticas observadas o realizadas por los practicantes. Enriquecer los análisis para superar opiniones superficiales y sostener las decisiones pedagógicas en argumentaciones bien informadas, con referentes teóricos diversos y concurrentes que ofrezcan mayor comprensión de lo que transcurre en el devenir cotidiano de las instituciones infantiles. Las teorías cumplen con la función de ayudar a de-velar, des-cubrir, comprender, y entonces contar con razones para decidir de acuerdo con los propósitos y principios explícitos que se sostengan.

En tanto eje vertebrador, desde la didáctica de la educación infantil se ha de establecer un "doble diálogo": uno hacia el interior de los espacios de formación docente entre aportes del campo de la formación específica, general y de las prácticas, y otro hacia el afuera, esto es, entre las instituciones de educación inicial y la institución formadora. ¿Qué pasaría si todos los profesores formadores incluyen en su bibliografía la ficha sobre "los pilares de la didáctica de la educación inicial? ¿Qué riqueza de lecturas podremos ofrecer a los estudiantes? Entonces nos correríamos de lógicas disciplinares para comprender cómo hacer aportes de los campos disciplinares a la didáctica de la educación infantil, desde donde se han de construir las configuraciones didácticas adecuadas para enseñar a niños pequeños.

En el campo de la formación específica, la didáctica de la educación infantil ofrece sus marcos teóricos propios en permanente construcción. En esta instancia formativa, se 
han de incluir bibliografías con potencialidad práctica, explicativa y problematizadora, para dar así la oportunidad de que el abordaje de la enseñanza sea desde diferentes dimensiones, con teorizaciones de los diferentes niveles que propone Ferry (1997). En el campo de la formación práctica, se ha de contribuir a la elaboración de saberes pedagógicos o de “certezas situadas", construidas a partir de escribir sus prácticas, tarea muy enriquecedora para que los estudiantes logren sus versiones propias de los repertorios de las formas de enseñar adecuadas a los niños pequeños. Propuestas como construir una carpeta de ruta, diario del practicante en el que por medio del acto de escritura se invita al estudiante a participar de procesos reflexivos que construyen formas de enseñar.

Estos son solo algunos lineamientos de un vasto repertorio de actividades, talleres y espacios formativos para poner en cuestión y ayudar a construir una mirada comprometida sobre la enseñanza en los primeros años, incluyendo la dimensión ética y política de la responsabilidad de educar, y ofrecer el acceso al mundo cultural a los niños pequeños.

\section{Síntesis}

Las cinco Propuestas para pensar juntos en las mejores formas de organizar la enseñanza para los niños pequeños son:

1. Tomar en cuenta los principios pedagógicos enunciados en los Pilares de la Didáctica de la Educación Infantil, los que responden a los interrogantes: qué, como, por qué y para qué enseñar.

2. Definir las actuaciones docentes a partir de las ocho formas de enseñar específicas cuando se trata de enseñar a niños menores de 6 años.

3. Diseñar la enseñanza, organizar intencionalmente lo que se ofrece pensando las diversas “configuraciones didácticas” propias de la Educación Inicial, como lo son las propuestas de juego, propuestas para conocer el ambiente, vida cotidiana, experiencias estéticas y multitarea.

4. Defender y reclamar que la enseñanza suceda en Escuelas infantiles en sentido amplio, en instituciones que asuman la responsabilidad de educar, no solo cuidar, no solo asistir, no solo estimular, sino educar en el sentido de hacer real el derecho a la inclusión y la calidad, a la educación para todos los niños y todas las niñas en tanto ciudadanos desde que nacen.

5. Enseñar a enseñar en los espacios de formación docente, en constante "diálogo" entre ellos y con los espacios de las prácticas, promoviendo el acceso a las experiencias de escritura de sus propias prácticas, lo cual permite construir teoría pedagógica personal, certezas situadas como referentes para la acción. 


\section{A modo de cierre y apertura}

Se propone pensar en el campo de la pedagogía infantil como un campo en construcción. La didáctica de la educación infantil, cuyo objeto de estudio es la enseñanza, forma parte de la pedagogía infantil, campo teórico en construcción al igual que el campo de la pedagogía de la crianza. Pensar en una pedagogía infantil ubica a la didáctica de la educación inicial como una de sus dimensiones, la encuadra en un marco político, social, histórico y pedagógico-didáctico rico en argumentaciones, que suman al reconocimiento de la identidad propia de la didáctica de la educación inicial como didáctica específica y necesaria que responde a las particulares formas de enseñar que demandan los niños pequeños.

\section{Referencias}

Bialostocki, P., Macías, M., Marotta, E., Moreno, P., Rebaglistti, S., Sena, C., y Violante, R. (2016). Debates y claves en la educación maternal hoy. Recuperado de https://drive.google.com/open?id=oBzIc3yoPeIQ4SHZpbmF1eG1wSTA

Penchansky de Bosch, L. y San Martin de Duprat, H. A. (1995). El nivel inicial. Estructuración. Orientaciones para la práctica. Buenos Aires: Colihue.

Sarlé, P., Rodríguez S., I. y Rodríguez de P., E. (2010). Juego. Fundamentos y reflexiones en torno a su enseñanza. Serie: El Juego en el Nivel Inicial. Propuestas de enseñanza. Cuadernillo N. ${ }^{\circ}$ 1. IDIE /OEI, Unicef, Fundación BBVA. Recuperado de http://files.unicef.org/argentina/spanish/Cuaderno_1_Fundamentos.pdf

Soto, C. y Violante, R. (2005). En el Jardín Maternal. Investigaciones y propuestas. Buenos Aires: Paidós.

Soto, C. y Violante, R. (2009) Didáctica de la Educación Inicial documento de apoyo curricular MC y E INFD 2009. Recuperado de http://repositorio. educacion.gov.ar/dspace/bitstream/handle/123456789/89819/Didactica\%20 inicial.pdf? sequence $=1$

Violante, R. (2001) Enseñanza en el nivel inicial I y II. Gobierno de la Ciudad de Buenos Aires (GCBA). Dirección de Currícula. Formación Docente 2001. Aportes para el debate curricular. Recuperado de http://studylib.es/doc/6335702/ ense\%C3\%Bianza-en-el-nivel-inicial-1-y-2

Violante, R. (2006). Cómo enseñar a los niños pequeños. Educrianza. Recuperado de https://es.slideshare.net/NadiaJorgelinaLopez/2006-educrianzacomo-ensear-a-los-nios-pequeos

Violante, R (2008) Por una Educación Integral Selección de fragmento del Cap. 1 ¿Por qué Pedagogía de la Crianza? En C. Soto y R. Violante, Pedagogía de la crianza. Un campo teórico en construcción. Buenos Aires: Paidós. 
Violante, R. (2011, septiembre). La centralidad del juego como uno de los pilares de la didáctica de la educación inicial. Conferencia Cátedra Abierta Juego. Ministerio Nacional de Educación. Dirección de Educación Inicial. Disponible en https:// drive.google.com/a/bue.edu.ar/file/d/oBx_irZGIWcSISDVZNEs2cmlpcW8/ view?usp=sharing

Violante, R. (2013a). La Multitarea y la construcción de escenarios como dispositivos pedagógicos privilegiados en la Educación Inicial. Conferencia. Congreso de Educación Inicial. Escuela Normal Superior (ENS) $\mathrm{N}^{\circ}$ 3, Rosario. Sept. 2013. Revista Aula, (3). Recuperado de https://drive.google.com/a/bue. edu.ar/file/d/oBx_irZGIWcSINzJaRDJobFFsdExxaVhsUXVhXoppbVN3bFY4/ view? usp=sharing

Violante, R. (2013b). El juego trabajo, o juego en rincones, o en sectores o rincones de actividades. Cambios y permanencias. e-Eccleston. Temas de Educación Infantil, 9(18). Recuperado de http://ieseccleston.caba.infd.edu.ar/sitio/upload/ Revista_18_final_1.pdf

Violante, R. y Soto, C. (2011). Didáctica de la Educación Inicial: Los Pilares. En Foro para la Educación Inicial. Encuentro Regional del Sur. Políticas de Enseñanza y definiciones curriculares. MC y E. Recuperado de https://drive.google.com/a/ bue.edu.ar/file/d/oBx_irZGIWcSIOHQ1QnliYWdlMXM/view?usp=sharing

Violante, R. y Soto, C. (2015). La organización de la enseñanza. Temas de inicial. Vol. 8. La organización de la enseñanza. Ministerio de Cultura y Educación. Recuperado de http://repositorio.educacion.gov.ar:8080/dspace/handle/123456789/110172

Violante, R. y Rodríguez de Pastorino, E. (2017). Encuadre Pedagógico-Didáctico para versión preliminar del diseño curricular para la educación inicial de la Provincia de Río Negro. Ministerio de Educación y Derechos Humanos. Dirección de Planeamiento, Educación Superior y Formación. Documento sin publicar.

Zabalza, M. (2000). Equidad y calidad en la educación Infantil. Una lectura desde el currículo. Ponencia en el Simposio Mundial de educación Infantil, Santiago de Chile. 